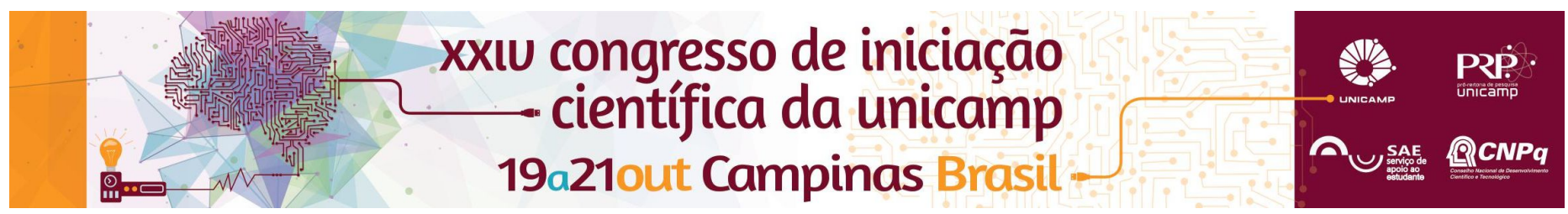

\title{
Sugarcane Transformation Protocol Standardization Using a Dry Resistance Gene
}

\section{Rafael H. Gallinari*, Giovanna V. Guidelli, Marcelo Menossi}

\begin{abstract}
There are several published works about the success in sugarcane transformation, with a wide range of possible methodologies. However, there are still difficulties in establishing an efficient and reproducible tissue culture techniques, being of great importance the establishment of an effective protocol for the transformation of sugarcane.
\end{abstract}

\section{Key words:}

Sugarcane, Transformation, Protocol

\section{Introduction}

The sugarcane (Saccharum spp.) is a culture of global economic importance, highlighting in the production of sugar and renewable energy. Thus, the development of new cultivars combining tools of classical breeding and biotechnology emerges as a promising alternative to increase the productivity of sugarcane without impacting the expansion of cultivation area.

Although there are studies reporting the success in sugar cane transformation, with a large number of methodologies (Singh et al., 2013), there are still difficulties in establishing tissue culture techniques for an efficient and reproducible protocol and being of great importance to establish an effective protocol for the transformation of sugarcane.

\section{Results and Discussion}

1. Tests performed for standardization of the protocol

\subsection{Distilled water $\times$ Citric acid solution to introduce pointers}

During the introduction of top-stalks at the moment of cutting the cane palmettos, it is normal that the explants oxidize slightly due to light. A strategy adopted is the use of citric acid solution, as it prevents this oxidation until the explants are transferred to the medium and left in the dark.

In this experiment, 25 pointers were introduced into citric acid solution and 27 pointers in distilled water, generating 50 and 54 plates respectively.

There were no significant differences in the degree of oxidation of explants (Img. 1), which allowed us to conclude that this type of selection there is no need to use citric acid in the introduction of explants. When used citric acid, there is the need for $\mathrm{pH}$ correction. As it was found that there were no differences in the addition of the same, this represents a saving of reagents and solutions preparation time.

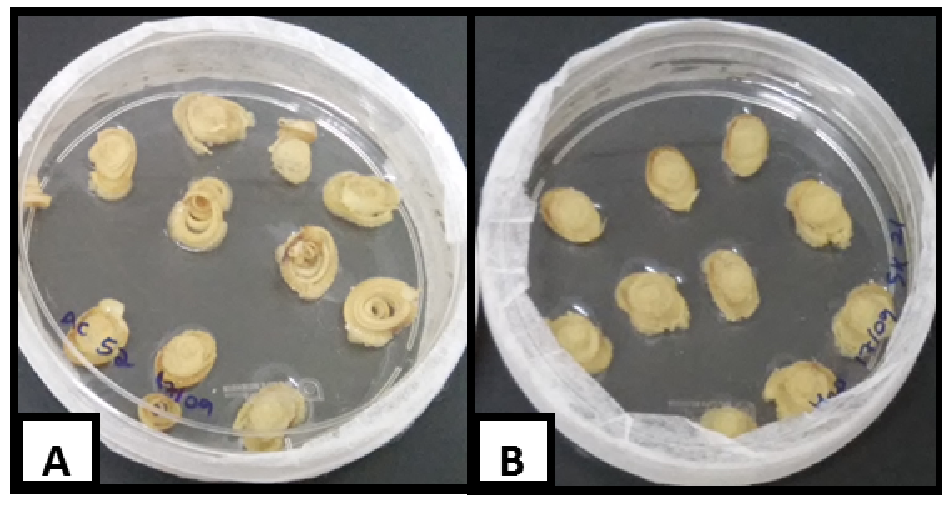

Image 1. Comparison of the material introduced using citric acid solution (A) and distilled water (B).

\subsection{Standardization of reagents in the preparation of culture medium}

In wild material stage was standardized the use of the same culture medium.

\section{Conclusions}

Although various tests have been conducted and a significant amount of procedures have been standardized for the wild materials tissue culture, still exists many possibilities that need to be set for the development and growth of embryogenic calli to occur effectively the transformation of the same.

The processes that have been standardized correspond to an initial protocol establishment step for allowing the processing of sugarcane throughout the laboratory.

\section{Acknowledgement}

This work was carried out with $\mathrm{CNPq}$ support, National Council for Scientific and Technological Development - Brazil

To the professor Marcelo Menossi, to Dr. Paulo Cezar De Lucca and a special thanks to the PhD student Giovanna Vieira Guidelli.

SINGH, R. K.; KUMAR, P.; TIWARI, N. N.; RASTOGI, J.;SINGH, S. P. Current Status of Sugarcane Transgenic: an Overview. Advancements in Genetic Engineering, Vol. 2, India. 2013. 